\title{
A Maximum-Principle-Preserving Third Order Finite Volume SWENO Scheme on Unstructured Triangular Meshes
}

\author{
Yunrui Guo, Lingyan Tang*, Hong Zhang and Songhe Song \\ Department of Mathematics and Systems Science, and State Key Laboratory of High \\ Performance Computing, National University of Defense Technology, Changsha, \\ Hunan 410073, China
}

Received 24 November 2016, Accepted (in revised version) 12 May 2017

\begin{abstract}
We modify the construction of the third order finite volume WENO scheme on triangular meshes and present a simplified WENO (SWENO) scheme. The novelty of the SWENO scheme is the less complexity and lower computational cost when deciding the smoothest stencil through a simple mechanism. The LU decomposition with iterative refinement is adopted to implement ill-conditioned interpolation matrices and improves the stability of the SWENO scheme efficiently. Besides, a scaling technique is used to circument the growth of condition numbers as mesh refined. However, weak oscillations still appear when the SWENO scheme deals with complex low density equations. In order to guarantee the maximum-principle-preserving (MPP) property, we apply a scaling limiter to the reconstruction polynomial without the loss of accuracy. A novel procedure is designed to prove this property theoretically. Finally, numerical examples for one- and two-dimensional problems are presented to verify the good performance, maximum principle preserving, essentially non-oscillation and high resolution of the proposed scheme.
\end{abstract}

AMS subject classifications: 65M10, 78A48

Key words: Triangular meshes, WENO, scaling limiter, maximum-principle-preserving.

\section{Introduction}

We consider the two-dimensional scalar conservation law

$$
\frac{\partial u}{\partial t}+\frac{\partial f(u)}{\partial x}+\frac{\partial g(u)}{\partial y}=0
$$

\footnotetext{
*Corresponding author.

Email: yunruiguo@nudt.edu.cn (Y. R. Guo)
} 
subject to the initial condition $u(x, y, 0)=u_{0}(x, y)$. WENO schemes are high order numerical methods for solving PDEs which may contain discontinuities, sharp gradient regions and other complex solution structures. The main idea of WENO schemes is to form a weighted combination of several local reconstructions based on different stencils and use it as the final WENO reconstruction. Thus WENO schemes have the ability to achieve high order accuracy in smooth regions while maintaining sharp and essentially monotone shock transitions.

Abgrall first recalled and improved the results of an earlier literature about nonoscillatory reconstruction on unstructured meshes in literature [1]. Later $\mathrm{Hu}$ and Shu [2] constructed high order weighted essentially non-oscillatory schemes on unstructured triangular meshes in the finite volume formulation. They presented third order schemes using a combination of linear polynomials and fourth order schemes using a combination of quadratic polynomials. However, oscillations would arise when strong discontinuities are included in the solution. In 2005, Haslbacher proposed a weighted essentially non-oscillatory (WENO) reconstruction algorithm. The stencils are fixed to reduce a high computational cost typically associated with the WENO scheme [3]. For accurate simulations of high Mach number aerodynamic flows with strong discontinuities, Wolf and Azevedo described the implementation and analysis of the high order ENO and WENO schemes applied to high speed flows on unstructured grids in [4]. The advantage of the method is that the reconstruction stencils can be composed by control volumes with any number of edges, e.g., triangles, quadrilaterals and hybrid meshes. Singular unstructured meshes always lead to ill-conditioned coefficient matrices. Therefore, a robust WENO reconstruction procedure was designed to deal with distorted local mesh geometries or degenerate cases when the mesh quality varies for complex domain geometry [5]. Besides, a new family of ENO schemes were derived via Taylor series expansion and solved using a weighted least squares formulation [6], which did not require constructing sub-stencils. Thus it provides a more flexible framework and less sensitivity to the mesh.

There are two types of finite volume WENO schemes on unstructured meshes in the literature above mentioned. The first type consists of WENO schemes whose orders are not higher than that of the reconstruction on each small stencil, such as $[6,7]$. For this type, the non-linear weights do not contribute to the increase of the order but to the nonlinear stability purely, or to avoid spurious oscillations. The second type consists of WENO schemes whose order is higher than that of the reconstruction on each small stencil, such as $[2,8]$. They are more complex than the former that only need to choose the linear weights as arbitrary positive numbers for the better linear stability. In this paper, we focus on the finite volume WENO scheme on unstructured triangular meshes [9] belonging to the first type. The simple strategy of the stencil construction can achieve the third order accuracy using less cells but higher resolution than other WENO schemes on triangular meshes $[6,11]$. A crucial step in building this scheme is to compute interpolation matrices. However, traditional solvers for linear systems are prone to collapse for ill-conditioned matrices, such as the LU decomposition, the Jacobi iterative method and the Gauss-Seidel 
method. We effectively improve the accuracy of the solution using a solver with iterative refinement. Besides, a scaling technique is used to circumvent the bad condition number due to mesh refinement.

The solution to the scalar conservation law (1.1) which has the MPP property indicates that, if the initial condition is bounded $m \leq u_{0} \leq M$, then $m \leq u(x, y, t) \leq M$ for all the future times $t>0$. As the components of the solutions to the compressible Euler system, both densities and pressures must be positive in each situation. The failure of preserving positivity of density or pressure may cause blow-ups, for instance, the low density problems of blast wave and low pressure problems in computing high Mach number astrophysical jets [12]. However, the SWENO scheme can not retain the MPP/PP property. Several methods have been adopted to handle such problems. A parameterized flux limiter was generalized to finite volume WENO schemes on unstructured meshes without excessively restricting the CFL condition [13]. Based on the main ideas of MPP/PP high order schemes, Zhang and Shu presented a simple implementation which result in a significant reduction of computational cost especially for WENO finite volume schemes [10]. Then they extended a scaling limiter to a general framework of constructing arbitrarily high order accurate maximum-principle-satisfying schemes for two-dimensional scalar conservation laws on triangular meshes [11]. Our main objective is to design a scaling limiter for the SWENO scheme to satisfy the strict maximum principles for the scalar conservation laws in two dimensions. Other works considering the MPP/PP property have been presented in [14].

The rest of the paper is organized as follows. Section 2 emphasizes on the construction of the SWENO finite volume scheme for the scalar conservation law in two dimensions. The LU decomposition with iterative refinement and the scaling technique are introduced in detail. In Section 3, a special quadrature rule over a triangle is presented for decomposing the cell average into a convex combination of the point values of the approximation polynomial. Then we introduce a scaling limiter and prove that the scaling limiter does not damage the accuracy by using a technique simpler than the work in [10,11]. At last, some important manipulation details are also described. In Section 4, the proposed scheme is tested for some very demanding numerical problems. Concluding remarks are given in Section 5.

\section{SWENO reconstruction on triangular meshes}

\subsection{The finite volume formulation on triangular meshes}

In this section, we consider the two-dimensional scalar conservation law (1.1) using the finite volume formulation. Taking the triangle as control volume, we formulate the semidiscrete finite volume scheme of Eq. (1.1) as

$$
\frac{d}{d t} \bar{u}_{T}(t)+\frac{1}{|T|} \int_{\partial T} F \cdot \vec{n} d s=0,
$$


where $\bar{u}_{T}(t)=\frac{1}{|T|} \int_{\partial T} u d s$ stands for the cell average on the cell $T, F=(f, g)$ and $\vec{n}$ is the outward unit normal vector of the triangle boundary $\partial T,|T|$ is the area of $T$.

The line integral in Eq. (2.1) is discretized by a $q$-point Gaussian integration formula,

$$
\int_{\partial T} F \cdot \vec{n} d s \approx \sum_{k=1}^{3}\left|\partial T_{k}\right| \sum_{\beta=1}^{q} w_{\beta} F\left(u\left(G_{\beta}^{k}\right)\right) \cdot \vec{n}_{k}
$$

where $\left|\partial T_{k}\right|$ is the length of the $k$-th side of $T, G_{j}^{k}$ and $w_{j}$ are the Gaussian quadrature points and weights respectively, and $F\left(u\left(G_{j}^{k}\right)\right) \cdot \vec{n}_{k}$ is approximated by a numerical flux. We apply the Lax-Friedrichs flux in all our numerical experiments, which is given by

$$
h\left(u^{-}, u^{+}, \vec{n}\right)=\frac{1}{2}\left[\left(F\left(u^{-}\right)+F\left(u^{+}\right)\right) \cdot \vec{n}-a\left(u^{+}-u^{-}\right)\right],
$$

where $a$ is taken as an upper bound for the magnitude of the eigenvalues of the Jacobian in the $\vec{n}_{k}$ direction, $u^{-}$and $u^{+}$are the values of $u$ inside the triangle and outside the triangle (inside the neighboring triangle) at the Gaussian points.

Considering that we construct a third order accuracy finite volume scheme, the twopoint Gaussian integration formula is used. For the edge with the endpoints $P_{1}$ and $P_{2}$, the Gaussian quadrature points are $G_{1}=c P_{1}+(1-c) P_{2}$ and $G_{2}=(1-c) P_{1}+c P_{2}$, where $c=\frac{1}{2}+\frac{\sqrt{3}}{2}$ and the Gaussian quadrature weights are $w_{1}=w_{2}=\frac{1}{2}$.

\subsection{Reconstruction of quadratic polynomials}

The key procedure for the SWENO scheme is to build a high order WENO reconstruction for the point values at the Gaussian quadrature points. For the construction of a $n$-th order polynomial, one must use $N(n)=\frac{(n+1)(n+2)}{2}$ cells or more. So every third order stencil should include at least 6 cells. Let the averaged value of each variable be given on every triangle; we will make use of these current conditions to construct a weighted quadratic polynomial on every cell. At first we select the smoothest second order stencil for each cell. Based on the second order stencil, we determine the cells required by constructing quadratic polynomials. The construction process is as follows:

In Fig. 1, $T_{1}$ is the center cell of the mesh. To construct a second order stencil including three cells, we can choose any two cells from $T_{2}, T_{3}$ and $T_{4}$. So three second order stencils can be obtained. Next we choose the smoothest one according to the magnitude of $I D_{i j}$ computed by

$$
I D_{i j}=\frac{\left|\bar{u}_{T_{1}}-\bar{u}_{T_{i}}\right|}{\left|T_{i}\right|}+\frac{\left|\bar{u}_{T_{1}}-\bar{u}_{T_{j}}\right|}{\left|T_{j}\right|}, \quad i \neq j ; \quad i, j=2,3,4 .
$$

However, the similar step is executed in [9] by constructing linear polynomials

$$
p_{k}^{1}(x, y)=a_{0}+a_{1} x+a_{2} y, \quad k=1,2,3,
$$




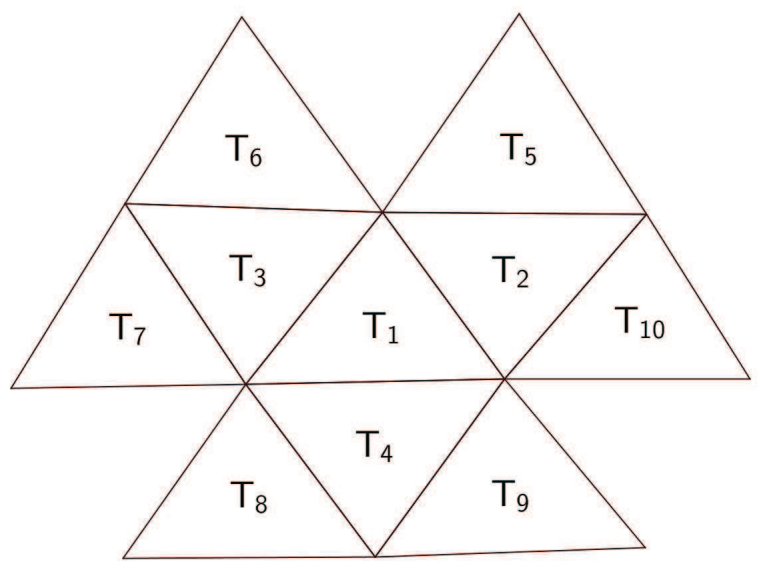

Figure 1: A typical mesh.

under the constraints

$$
\frac{1}{\left|T_{i}\right|} \int_{T_{i}} p_{k}^{1}(x, y)=\bar{u}_{T_{i}}
$$

Then let $I D_{i j}$ be equal to $\left|a_{1}\right|+\left|a_{2}\right|$. For every cell in each time stage, we need to solve three linear systems, which cause a huge computational cost.

For convenience, we assume that the second order stencil $\left\{T_{1}, T_{2}, T_{3}\right\}$ is the smoothest. To construct a quadratic polynomial on the cell $T_{1}$, three additional cells in the neighborhood of the cell $T_{1}$ are required. Since $T_{5}, T_{10}$ and $T_{6}, T_{7}$ have common edges with $T_{2}$ and $T_{3}$ respectively, $T_{4}$ has a common edge with $T_{1} . T_{8}, T_{9}$ which are close to $T_{4}$ may become steeper than $T_{5}, T_{10}, T_{6}$ and $T_{7}$. Add $T_{4}$ and any two cells from $T_{5}, T_{6}, T_{7}, T_{10}$ to the second order stencil consisting of $T_{1}, T_{2}, T_{3}$. Thus we can obtain 6 composed stencils $S_{k}(k=1,2, \cdots, 6)$ with 6 quadratic polynomials as following:

$$
\begin{array}{ll}
p_{1}^{2}(x, y): S_{1}=\left\{T_{1}, T_{2}, T_{3}, T_{4}, T_{5}, T_{6}\right\}, & p_{2}^{2}(x, y): S_{2}=\left\{T_{1}, T_{2}, T_{3}, T_{4}, T_{5}, T_{7}\right\}, \\
p_{3}^{2}(x, y): S_{3}=\left\{T_{1}, T_{2}, T_{3}, T_{4}, T_{5}, T_{10}\right\}, & p_{4}^{2}(x, y): S_{4}=\left\{T_{1}, T_{2}, T_{3}, T_{4}, T_{6}, T_{7}\right\}, \\
p_{5}^{2}(x, y): S_{5}=\left\{T_{1}, T_{2}, T_{3}, T_{4}, T_{6}, T_{10}\right\}, & p_{6}^{2}(x, y): S_{6}=\left\{T_{1}, T_{2}, T_{3}, T_{4}, T_{7}, T_{10}\right\},
\end{array}
$$

where $p_{k}^{2}(x, y), k=1,2, \cdots, 6$, are quadratic polynomials under the constraints

$$
\left\langle p_{T}^{2}(x, y)\right\rangle_{T_{i}}:=\frac{1}{\left|T_{i}\right|} \int_{T_{i}} p_{k}^{2}(x, y)=\bar{u}_{T_{i}}, \quad i=1,2, \cdots, 6 .
$$

Denote the smoothness of $p_{k}^{2}(x, y)$ as $I S_{k}$, which is the summation of all square values of average difference between every two cells with a common edge in the stencil [9]. If $u$ is smooth on the stencil $S_{k}, I S_{k}=\mathcal{O}\left(h^{2}\right)$ ( $h$ is the maximum radius of the circumcircle of the cells); if $u$ is discontinuous on the stencil $S_{k}$, then $I S_{k}=\mathcal{O}(1)$. 
Let

$$
\beta_{k}=\frac{\alpha_{k}}{\sum_{i=1}^{6} \alpha_{i}}, \quad \alpha_{k}=\frac{1}{\left(\epsilon+I S_{k}\right)^{2}},
$$

where $\epsilon$ is a positive real number which prevents the denominator from becoming zero (In our numerical tests, $\epsilon$ is taken as $10^{-5}$ ).

Thus we gain the final weighted quadratic polynomial

$$
p_{T_{1}}^{2}(x, y)=\sum_{k=1}^{6} \beta_{k} \cdot p_{k}^{2}(x, y)
$$

In smooth regions,

$$
\beta_{k}=\frac{\alpha_{k}}{\sum_{i=1}^{6} \alpha_{i}}=\mathcal{O}(1)
$$

We observe that the interpolating polynomials contribute to the weighted quadratic polynomial. While in discontinuous regions,

$$
\beta_{k}=\frac{\alpha_{k}}{\sum_{i=1}^{6} \alpha_{i}}=\frac{\frac{1}{(\epsilon+\mathcal{O}(1))^{2}}}{\sum_{i=1}^{6} \alpha_{i}}=\frac{\mathcal{O}(1)}{\sum_{i=1}^{6} \alpha_{i}} \leq \max \left(\mathcal{O}\left(\epsilon^{2}\right),\left(h^{4}\right)\right),
$$

which hardly contribute to the weighted quadratic polynomial.

\subsection{Efficient implementation of ill-conditioned interpolation matrix}

Give a standard representation of $p_{T}^{2}(x, y)$ :

$$
p_{T}^{2}(x, y)=\sum_{l=0}^{2} \sum_{k+j=l} a_{k j} x^{k} y^{j}
$$

Let all Eq. (2.5) except for the case $i=1$ minus the first equation, then the linear system for the reconstruction polynomial can be written in a compact form:

$$
B A=\bar{U},
$$

where

$$
\begin{aligned}
B & :=\left[\left\langle x^{k} y^{j}\right\rangle_{T_{i}}-\left\langle x^{k} y^{j}\right\rangle_{T_{1}}\right] \\
& =\left[\begin{array}{cccc}
\langle x\rangle_{T_{2}}-\langle x\rangle_{T_{1}} & \langle y\rangle_{T_{2}}-\langle y\rangle_{T_{1}} & \cdots & \left\langle y^{2}\right\rangle_{T_{2}}-\left\langle y^{2}\right\rangle_{T_{1}} \\
\vdots & \vdots & \vdots & \vdots \\
\langle x\rangle_{T_{6}}-\langle x\rangle_{T_{1}} & \langle y\rangle_{T_{6}}-\langle y\rangle_{T_{1}} & \cdots & \left\langle y^{2}\right\rangle_{T_{6}}-\left\langle y^{2}\right\rangle_{T_{1}}
\end{array}\right], k+j=l, 1 \leq l \leq 2, i=2, \cdots, 6,
\end{aligned}
$$


is a $5 \times 5$ interpolation matrix and

$$
\begin{aligned}
& A:=\left[a_{10}, a_{01}, a_{11}, a_{20}, a_{02}\right]^{T}, \\
& \bar{U}:=\left[\bar{u}_{T_{2}}-\bar{u}_{T_{1}}, \bar{u}_{T_{3}}-\bar{u}_{T_{1}}, \bar{u}_{T_{4}}-\bar{u}_{T_{1}}, \bar{u}_{T_{5}}-\bar{u}_{T_{1}}, \bar{u}_{T_{6}}-\bar{u}_{T_{1}}\right]^{T} .
\end{aligned}
$$

It is a mathematically uniquely solvable problem to compute $A$ if $S$ is an admissible stencil, but in practice one encounters two serious difficulties:

- The integral operation of matrix entries are trivial and expensive.

- The matrix $B$ may have a bad condition number, which disturbs the accuracy and stability.

For the first problem, the elements of the matrix $B$ need to be computed once and stored for later use. Using the formula (2.8), we calculate the integrals

$$
\left\langle x^{k} y^{j}\right\rangle_{T^{\prime}} \quad k+j=l, \quad 0 \leq l \leq 2,
$$

and store them for each cell $T$, these wouldn't request too much memory.

For the second problem, there are two possible reasons leading to a bad condition number of $B$ : first, the condition number grows like $h^{-n}$ when computational meshes are refined [1]; second, the geometric behaviors of the components in the stencil $S_{k}$ can influence the condition number of $B$.

The first reason can be circumvented by replacing the representation of the polynomial $p_{k}^{2}(x, y)$ by barycentric coordinates [1]. Here we introduce a simpler technique to obtain a condition number independent from $h$. Define a local scaling factor

$$
s:=\frac{1}{\sqrt{|T|}}
$$

which approximates $1 / h$ and changes the standard representation (2.8) into

$$
p_{T}^{2}(x, y)=\sum_{l=0}^{2} \sum_{k+j=l} s^{l} \tilde{a}_{k j} x^{k} y^{j},
$$

the interpolation matrix $B$ becomes

$$
\tilde{B}:=\left[s^{l}\left\langle x^{k} y^{j}\right\rangle_{T_{i}}-s^{l}\left\langle x^{k} y^{j}\right\rangle_{T_{1}}\right], \quad k+j=l, \quad 0 \leq l \leq 2, \quad i=2, \cdots, 6,
$$

we get the standard representation (2.8) easily from $A=\left(s \tilde{a}_{10}, s \tilde{a}_{01}, s^{2} \tilde{a}_{11}, s^{2} \tilde{a}_{20}, s^{2} \tilde{a}_{02}\right)^{T}$, more details refer to [7].

The second reason cannot be circumvented easily. We adopt the LU decomposition with iterative refinement [15] to improve the solutions of ill-conditioned linear systems. The flowchart is as follows: 
Step 1 Execute an LU decomposition (Doolittles method) of $\tilde{B}^{T}$ with partial pivoting:

$$
P \tilde{B}^{T} \approx U^{T} L^{T},
$$

then we solve

$$
L U P A=\bar{U}
$$

and obtain

$$
\tilde{A} \approx P^{T} U^{-1} L^{-1} \bar{U}
$$

Step 2 Calculate the residual

$$
r_{k}=\bar{U}-\tilde{B} \tilde{A}_{k}
$$

and solve

$$
\tilde{B} x=r_{k}
$$

using the LU decomposition obtained at Step 1.

Let $x_{k}$ be an approximate solution of Eq. (2.10). Then we update $\tilde{A}_{k}$ by

$$
\tilde{A}_{k+1}=\tilde{A}_{k}+x_{k} \text {. }
$$

Repeat the process until the following conditions are satisfied:

$$
\frac{\left\|\tilde{A}_{k+1}-\tilde{A}_{k}\right\|}{\left\|\tilde{A}_{k+1}\right\|}<\epsilon .
$$

Generally, $\epsilon$ is taken from $10^{-8} \sim 10^{-13}$.

- The number of iterations reaches $k_{\max }$, then one has to skip this stencil.

The solution obtained via this method is normally more accurate than one by pure floating-point arithmetic in the working precision, though it depends on the matrix.

\subsection{Temporal discretization}

The semi-discrete Eq. (2.1) is a system of ODEs. Let the time step size be $\Delta t$. We use the third order TVD Runge-Kutta method (also referred to as the strong stability preserving (SSP) third order time discretization) of Shu and Osher [16] to achieve the same accuracy order in both time and space direction. The method is given as

$$
\begin{aligned}
& u_{T}^{(1)}=u_{T}^{n}+\Delta t F\left(u_{T}^{n}\right), \\
& u_{T}^{(2)}=\frac{3}{4} u_{T}^{n}+\frac{1}{4}\left(u_{T}^{(1)}+\Delta t F\left(u_{T}^{(1)}\right)\right), \\
& u_{T}^{n+1}=\frac{1}{3} u_{T}^{n}+\frac{2}{3}\left(u_{T}^{(2)}+\Delta t F\left(u_{T}^{(2)}\right)\right),
\end{aligned}
$$

where $F(u)$ is a spatial operator, which satisfies the MPP/PP property. Since a SSP high order time discretization is a convex combination of the Euler forward, the full scheme with a high order SSP time discretization will still satisfy the maximum principle [11]. 


\section{Scaling limiter and its implementation for the SWENO scheme}

The Euler forward time discretization has the MPP property for the two-dimensional scalar conservation laws on triangular meshes. This result has been proven completely in literature [11]. Firstly a special quadrature rule which is exact for a given degree twovariable polynomial over a triangle is introduced. Then the cell average is decomposed into a convex combination of the point values of the approximation polynomial, including all the Gauss quadrature points for each edge. Based on this result, we can obtain a sufficient condition to ensure that the Euler forward time discretization satisfies a stric$t$ maximum principle on triangular meshes. Actually, the above proof can be achieved with the same procedure but the less Gauss quadrature points for the proposed third order scheme. Considering the spatial discretization (2.1) in Section 2, the scheme with the Euler forward time discretization is given by

$$
\bar{u}_{T}^{n+1}=\bar{u}_{T}^{n}-\frac{\Delta t}{|T|} \sum_{k=1}^{3}\left|\partial T_{k}\right| \sum_{\beta=1}^{2} w_{\beta} h\left(u^{-}\left(G_{\beta}^{k}\right), u^{+}\left(G_{\beta}^{k}\right), \vec{n}_{k}\right),
$$

where the numerical flux function $h\left(u^{-}, u^{+}, \vec{n}\right)$ satisfies the conservativity, consistency and monotonicity. We plan to rewrite the right hand of (3.1) as a monotone increasing function of some point values of $p_{T}^{2}(x, y)$ under a certain CFL condition in the following subsections.

\subsection{The decomposition of the cell average}

We are interested in a quadrature rule on a triangle satisfying:

- The quadrature rule is exact for the integration of $p_{T}^{2}(x, y)$ on the cell $T$.

- All the quadrature weights should be positive.

- The quadrature points should include all the Gauss quadrature points on each edge $\partial T_{k}$.

For convenience, we denote the three vertices of the cell $T$ by the vectors $V_{1}, V_{2}$ and $V_{3}$, whose orientation is clockwise. The position vector $P$ of an arbitrary point in $T$ can be specified by the barycentric coordinates $\left(\beta_{1}, \beta_{2}, \beta_{3}\right)$, where $P=\beta_{1} V_{1}+\beta_{2} V_{2}+\beta_{3} V_{3}$. Consider the quadrature rule on the unit square $R:\left[-\frac{1}{2}, \frac{1}{2}\right]^{2}$ in the $u-v$ plane. Let $N$ be the smallest integer such that $2 N-3 \geq n$ ( $n$ is the degree of reconstruction polynomials), then the $N$-point Gauss-Lobatto quadrature rule is exact for a single variable polynomial of degree $n$ and the two endpoints are included in the $N$ quadrature points. We consider the case of $n=2$ and $N=3$ in this paper. Let $\left\{v_{\beta}: \beta=1,2\right\}$ denote the 2-point Gauss quadrature points on $R:\left[-\frac{1}{2}, \frac{1}{2}\right]$ with weights $w_{\beta}$, and $\left\{u_{\alpha}: \alpha=1,2,3\right\}$ denote the Gauss-Lobatto 
quadrature points on $R:\left[-\frac{1}{2}, \frac{1}{2}\right]$ with weights $\tilde{w}_{\alpha}$. And the two variables polynomial integral formula on $R$ can be got from the tensor product of the 3-point Gauss-Lobatto quadrature for $u$ and the 2-point Gauss quadrature for $v$, then the quadrature points can be written as $S_{R}=\left\{\left(u^{\alpha}, v^{\beta}\right): \alpha=1,2,3 ; \beta=1,2\right\}$. This quadrature is exact for a polynomial $p(u, v)$ if the degrees of $u$ and $v$ are less than $n$ and $2 n+1$ respectively [11]. Define the projections from the square $R$ to the triangle $T$ as:

$$
\begin{aligned}
& g_{1}(u, v)=\left(\frac{1}{2}+v\right) V_{1}+\left(\frac{1}{2}+u\right)\left(\frac{1}{2}-v\right) V_{2}+\left(\frac{1}{2}-u\right)\left(\frac{1}{2}-v\right) V_{3}, \\
& g_{2}(u, v)=\left(\frac{1}{2}+v\right) V_{2}+\left(\frac{1}{2}+u\right)\left(\frac{1}{2}-v\right) V_{3}+\left(\frac{1}{2}-u\right)\left(\frac{1}{2}-v\right) V_{1}, \\
& g_{3}(u, v)=\left(\frac{1}{2}+v\right) V_{3}+\left(\frac{1}{2}+u\right)\left(\frac{1}{2}-v\right) V_{1}+\left(\frac{1}{2}-u\right)\left(\frac{1}{2}-v\right) V_{2} .
\end{aligned}
$$

The above formulas map the quadrature points into a triangle. Therefore, we can use $g_{k}(u, v)$ and $S_{R}$ to construct the quadrature on the triangle $T$ :

$$
\bar{u}_{T}=\frac{1}{|T|} \int_{T} p_{T}^{2}(x, y) d s=\frac{1}{|T|} \int_{-\frac{1}{2}}^{\frac{1}{2}} \int_{-\frac{1}{2}}^{\frac{1}{2}} p_{T}^{2}\left(g_{k}(u, v)\right)\left|\frac{\partial g_{k}(u, v)}{\partial(u, v)}\right| d u d v, \quad k=1,2,3 .
$$

The Jacobian

$$
\left|\frac{\partial g_{k}(u, v)}{\partial(u, v)}\right|=2|T|\left(\frac{1}{2}-v\right) \geq 0,
$$

since the orientation of the three vertices is clockwise. We notice the integrand $p_{T}^{2}\left(g_{k}(u, v)\right)\left|\frac{\partial g_{k}(u, v)}{\partial(u, v)}\right|$ whose degrees of $u$ and $v$ are smaller than $n$ and $2 n+1$ respectively, thus the double integral in $u$ and $v$ is equal to the quadrature on $S_{R}$ :

$$
\bar{u}_{T}=\frac{1}{|T|} \int_{T} p_{T}^{2}(x, y) d s=\frac{1}{3} \sum_{k=1}^{3} \sum_{\alpha=1}^{3} \sum_{\beta=1}^{2} 2 p_{T}^{2}\left(g_{k}\left(u^{\alpha}, v^{\beta}\right)\right)\left(\frac{1}{2}-v^{\beta}\right) \tilde{w}_{\alpha} w_{\beta} .
$$

Denote the values of $p_{T}^{2}(x, y)$ at the quadrature points on the edge $\partial T_{k}$ as $u_{k, \beta}^{-}$. There are 6 quadrature points lying in the interior of $T$. Let the values of $p_{T}^{2}(x, y)$ at the quadrature points be $u_{\gamma}^{\text {int }}(\gamma=1, \cdots, 6)$ with the coefficients $w_{\gamma}^{\text {int }}$ in the decomposition. Then we can rewrite (3.3) as a convex combination of $u_{\gamma}^{\text {int }}$ and $u_{k, \beta}^{-}$:

$$
\bar{u}_{T}^{n}=\sum_{k=1}^{3} \sum_{\beta=1}^{2} \frac{2}{3} w_{\beta} \tilde{w}_{1} u_{k, \beta}^{-}+\sum_{\gamma=1}^{6} w_{\gamma}^{\text {int }} u_{\gamma}^{\text {int }} .
$$

However, the formula (3.4) for the proposed third order scheme in [11] consists of 9 points on the edges and 9 points in the triangle, which leads to a more trivial manipulation for the scaling limiter implementation. According to above decomposition, we can rewrite Eq. (3.1) as:

$$
\bar{u}_{T}^{n+1}=\sum_{k=1}^{3} \sum_{\beta=1}^{2} \frac{2}{3} w_{\beta} \tilde{w}_{1} u_{k, \beta}^{-}+\sum_{\gamma=1}^{6} w_{\gamma}^{i n t} u_{\gamma}^{i n t}-\frac{\Delta t}{|T|} \sum_{\beta=1}^{q} w_{\beta}\left(\sum_{k=1}^{3}\left|\partial T_{k}\right| h\left(u_{k, \beta}^{-}, u_{k, \beta}^{+}, \vec{n}_{k}\right)\right) .
$$


Lemma 3.1. Writing the right hand of (3.5) as a function $H$ of $u_{k, \beta^{\prime}}^{-} u_{k, \beta}^{+}$and $u_{\gamma}^{\text {int }}$,

$$
\bar{u}_{T}^{n+1}=H\left(u_{1,1}^{-}, u_{2,1}^{-}, \cdots, u_{3,2}^{-}, u_{1,1}^{+}, \cdots, u_{3,2}^{+}, u_{1}^{\text {int }}, \cdots, u_{6}^{\text {int }}\right),
$$

then $H$ is monotone increasing with respect to each argument, under the CFL condition

$$
a \frac{\Delta t}{|T|} \sum_{k=1}^{3}\left|\partial T_{k}\right| \leq \frac{2}{3} \tilde{w}_{1}
$$

where $\tilde{w}_{1}$ is the quadrature weight of the 3-point Gauss-Lobatto rule on $\left[-\frac{1}{2}, \frac{1}{2}\right]$.

The simple proof has been achieved in paper [11].

Next, we present a theorem:

Theorem 3.1. If the scheme (3.1) with the polynomial $p_{T}^{2}(x, y)$ satisfies the maximum principe

$$
m \leq \bar{u}_{T}^{n+1} \leq M,
$$

a sufficient condition is that

$$
m \leq u_{\gamma}^{\text {int }}, \quad u^{-}\left(G_{\beta}^{k}\right), u^{+}\left(G_{\beta}^{k}\right) \leq M,
$$

under the CFL constraint (3.6).

Proof. For an arbitrary constant $z$, the flux term inside the bracket of Eq. (3.5) satisfies

$$
\sum_{k=1}^{3}\left|\partial T_{k}\right| h\left(z, z, \vec{n}_{k}\right)=\sum_{k=1}^{3}\left|\partial T_{k}\right|(f(z), g(z)) \cdot \vec{n}_{k}=0,
$$

here the conservativity of the numerical flux is used. And the detailed proof is presented at the end of this section. According to the formulation (3.5), we have $H(z, \cdots, z)=z$. Then the monotonicity of $H$ in Lemma 3.1 promises that

$$
m=H(m, m, \cdots, m) \leq \bar{u}_{T}^{n+1} \leq H(M, M, \cdots, M)=M .
$$

Thus, we complete the proof.

Remark 3.1. Let $V_{1}=\left(x_{1}, y_{1}\right), V_{2}=\left(x_{2}, y_{2}\right), V_{3}=\left(x_{3}, y_{3}\right)$ and $x_{1}<x_{2}<x_{3}$. We denote the three edges of the triangle by the vectors

$$
E_{1}=\left(x_{3}-x_{1}, y_{3}-y_{1}\right), \quad E_{2}=\left(x_{3}-x_{2}, y_{3}-y_{2}\right), \quad E_{3}=\left(x_{2}-x_{1}, y_{2}-y_{1}\right),
$$

whose length are $\left|\partial T_{1}\right|,\left|\partial T_{2}\right|$ and $\left|\partial T_{3}\right|$ respectively. Thus their outward unit normal vectors can be indicated as

$$
\vec{n}_{1}=\frac{1}{\left|\partial T_{1}\right|}\left(y_{3}-y_{1}, x_{1}-x_{3}\right), \quad \vec{n}_{2}=\frac{1}{\left|\partial T_{2}\right|}\left(y_{3}-y_{2}, x_{2}-x_{3}\right), \quad \vec{n}_{3}=\frac{1}{\left|\partial T_{3}\right|}\left(y_{2}-y_{1}, x_{1}-x_{2}\right) .
$$

Plugging (3.9) into (3.7), we get the formula (3.8) easily. 


\subsection{Scaling limiter}

To enforce the sufficient condition in previous theorem, we need to modify the weighted quadratic polynomial $p_{T}^{2}(x, y)$, such that $p_{T}^{2}(x, y) \in[m, M]$ for all $(x, y) \in T$. For all $T$, assume that $\bar{u}_{T}^{n} \in[m, M]$, we use the following modified polynomial

$$
\tilde{p}_{T}^{2}(x, y)=\theta\left(p_{T}^{2}(x, y)-\bar{u}_{T}^{n}\right)+\bar{u}_{T}^{n}, \quad \theta=\min \left\{\left|\frac{M-\bar{u}_{T}^{n}}{M_{T}-\bar{u}_{T}^{n}}\right|,\left|\frac{m-\bar{u}_{T}^{n}}{m_{T}-\bar{u}_{T}^{n}}\right|, 1\right\},
$$

with

$$
M_{T}=\max \left\{\max _{k, \beta}\left\{u^{-}\left(G_{\beta}^{k}\right)\right\}, \max _{\gamma}\left\{u_{\gamma}^{\text {int }}\right\}\right\}, \quad m_{T}=\min \left\{\min _{k, \beta}\left\{u^{-}\left(G_{\beta}^{k}\right)\right\}, \min _{\gamma}\left\{u_{\gamma}^{\text {int }}\right\}\right\},
$$

instead of the definitions in [11]

$$
M_{T}=\max _{(x, y) \in T} p_{T}^{2}(x, y), \quad m_{T}=\min _{(x, y) \in T} p_{T}^{2}(x, y) .
$$

Thus $\tilde{p}_{T}^{2}(x, y)$ satisfies the following properties:

- Conservativity:

$$
\frac{1}{|T|} \int_{T} \tilde{p}_{T}^{2}(x, y) d x d y=\bar{u}_{T}^{n}
$$

- Accuracy: for smooth regions,

$$
\tilde{p}_{T}^{2}(x, y)-p_{T}^{2}(x, y)=\mathcal{O}\left(h^{3}\right), \quad \forall(x, y) \in T .
$$

The conservativity is straightforward, we are interested in the accuracy. Considering

$$
\theta=\left|\frac{M-\bar{u}_{T}^{n}}{M_{T}-\bar{u}_{T}^{n}}\right|
$$

and a non-constant polynomial $p_{T}^{n}(x, y)$,

$$
\tilde{p}_{T}^{2}(x, y)-p_{T}^{2}(x, y)=(\theta-1)\left(p_{T}^{2}(x, y)-\bar{u}_{T}^{n}\right)=\frac{M-\bar{u}_{T}^{n}}{M_{T}-\bar{u}_{T}^{n}}\left(p_{T}^{2}(x, y)-\bar{u}_{T}^{n}\right) .
$$

By the definition of $\theta$ in Eq. (3.10),

$$
\theta=\left|\frac{M-\bar{u}_{T}^{n}}{M_{T}-\bar{u}_{T}^{n}}\right|<1 \text {, i.e., } M_{T}>M \geq \bar{u}_{T}^{n} \geq m_{T} .
$$

Therefore, $p_{T}^{2}(x, y)$ is an approximation with the error $h^{3}$ of both $M_{T}$ and $M$, thus $M-\bar{u}_{T}^{n}=$ $\mathcal{O}\left(h^{3}\right), M_{T}-\bar{u}_{T}^{n}=\mathcal{O}\left(h^{3}\right)$. Since $p_{T}^{2}(x, y)-\bar{u}_{T}^{n}=\mathcal{O}\left(h^{3}\right)$, we have

$$
\tilde{p}_{T}^{2}(x, y)-p_{T}^{2}(x, y)=\frac{M-\bar{u}_{T}^{n}}{M_{T}-\bar{u}_{T}^{n}}\left(p_{T}^{2}(x, y)-\bar{u}_{T}^{n}\right)=\frac{\mathcal{O}\left(h^{6}\right)}{\mathcal{O}\left(h^{3}\right)}=\mathcal{O}\left(h^{3}\right) .
$$

We need to use the limiter in each stage for every weighted quadratic polynomial $p_{T}^{2}(x, y)$ in the third order Runge-Kutta method. At the same time, the CFL condition should be satisfied. Table 1 lists the barycentric coordinates of the quadrature points, which are used to compute $M_{T}$ and $m_{T}$. 
Table 1: The barycentric coordinates of the quadrature points.

\begin{tabular}{|c|c|c|c|}
\hline \multicolumn{2}{|c|}{ The quadrature points on the edges $u_{k, \beta}^{-}$} & \multicolumn{2}{|c|}{ The quadrature points in the triangle $u_{\gamma}^{\text {int }}$} \\
\hline$\left(\frac{1}{2}+\frac{\sqrt{3}}{2}, \frac{1}{2}-\frac{\sqrt{3}}{2}, 0\right)$ & $\left(\frac{1}{2}-\frac{\sqrt{3}}{2}, \frac{1}{2}+\frac{\sqrt{3}}{2}, 0\right)$ & $\left(\frac{1}{2}+\frac{\sqrt{3}}{2}, \frac{1}{2}-\frac{\sqrt{3}}{2}, \frac{1}{2}\right)$ & $\left(\frac{1}{2}-\frac{\sqrt{3}}{2}, \frac{1}{2}+\frac{\sqrt{3}}{2}, \frac{1}{2}\right)$ \\
\hline$\left(\frac{1}{2}+\frac{\sqrt{3}}{2}, 0, \frac{1}{2}-\frac{\sqrt{3}}{2}\right)$ & $\left(\frac{1}{2}-\frac{\sqrt{3}}{2}, 0, \frac{1}{2}+\frac{\sqrt{3}}{2}\right)$ & $\left(\frac{1}{2}+\frac{\sqrt{3}}{2}, \frac{1}{2}, \frac{1}{2}-\frac{\sqrt{3}}{2}\right)$ & $\left(\frac{1}{2}-\frac{\sqrt{3}}{2}, \frac{1}{2}, \frac{1}{2}\right.$ \\
\hline$\left(0, \frac{1}{2}+\frac{\sqrt{3}}{2}, \frac{1}{2}-\frac{\sqrt{3}}{2}\right)$ & $\left(0, \frac{1}{2}-\frac{\sqrt{3}}{2}, \frac{1}{2}+\frac{\sqrt{3}}{2}\right)$ & $\left(\frac{1}{2}, \frac{1}{2}+\frac{\sqrt{3}}{2}, \frac{1}{2}-\frac{\sqrt{3}}{2}\right)$ & $\left(\frac{1}{2}, \frac{1}{2}-\frac{\sqrt{3}}{2}, \frac{1}{2}\right.$ \\
\hline
\end{tabular}

\section{$4 \quad$ Numerical tests}

In this section, we test the proposed scheme on two-dimensional triangular meshes. Firstly, a linear equation, a Burgers equation and a smooth vortex problem are used to verify the numerical convergence and measure the convergence orders by the discrete $L_{1}$ and $L_{\infty}$ errors. Then we test the ability of the scheme to capture the shocks for Riemann problems of the compressible Euler equations. Finally, the two-dimensional Buckley-Leverett equation, the kinematic frontogenesis problem and the double mach reflection are solved. By comparing with other schemes we demonstrate the higher resolution of the SWENO scheme with the scaling limiter. The uniform triangular meshes shown in Fig. 2 are easy to refine, thus we use them to measure the convergence orders. The same effect can be achieved on non-uniform triangular meshes, shown in Fig. 3.

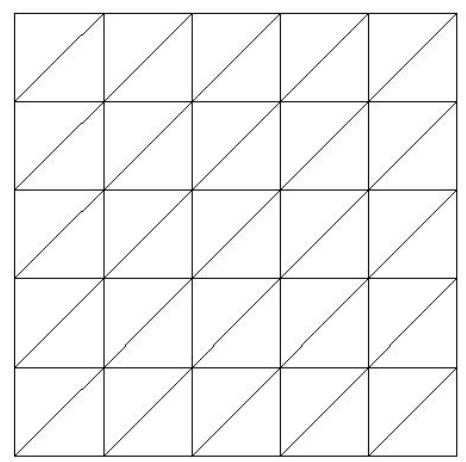

Figure 2: Uniform mesh.

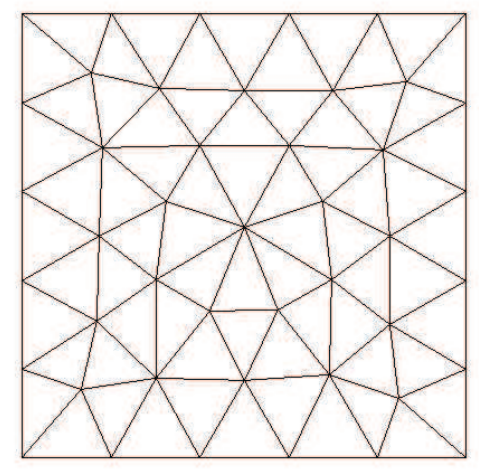

Figure 3: Non-uniform mesh.

\subsection{Two-dimensional linear equation}

To test the effectiveness of the proposed scheme, we first solve a two-dimensional linear wave equation:

$$
\left\{\begin{array}{l}
u_{t}+u_{x}+u_{y}=0 \\
u_{0}(x, y)=u(x, y, 0)
\end{array}\right.
$$


Table 2: Accuracy for 2D linear equation with scaling limiter.

\begin{tabular}{||c|cc|cc||}
\hline & \multicolumn{4}{|c||}{$u_{0}(x, y)=\sin (\pi(x+y))$} \\
\hline$N$ & $L_{1}$ error & order & $L_{\infty}$ error & order \\
\hline 5 & 0.5314 & $\cdots$ & 0.8296 & $\cdots$ \\
10 & 0.1842 & 1.5285 & 0.3000 & 1.4674 \\
20 & $2.155 \times 10^{-2}$ & 3.0955 & $3.938 \times 10^{-2}$ & 2.9294 \\
40 & $2.495 \times 10^{-3}$ & 3.1106 & $5.156 \times 10^{-3}$ & 2.9331 \\
80 & $3.139 \times 10^{-4}$ & 2.9907 & $8.137 \times 10^{-4}$ & 2.6637 \\
\hline
\end{tabular}

Table 3: Accuracy for 2D linear equation without scaling limiter.

\begin{tabular}{||c|cc|cc||}
\hline & \multicolumn{4}{|c||}{$u_{0}(x, y)=\sin (\pi(x+y))$} \\
\hline$N$ & $L_{1}$ error & order & $L_{\infty}$ error & order \\
\hline 5 & 0.5273 & $\cdots$ & 0.8274 & $\cdots$ \\
10 & 0.1724 & 1.6128 & 0.2124 & 1.9618 \\
20 & $2.112 \times 10^{-2}$ & 3.0291 & $3.777 \times 10^{-2}$ & 2.4915 \\
40 & $2.490 \times 10^{-3}$ & 3.0844 & $5.162 \times 10^{-3}$ & 2.8712 \\
80 & $3.194 \times 10^{-4}$ & 2.9627 & $8.067 \times 10^{-4}$ & 2.6778 \\
\hline
\end{tabular}

with periodic boundary conditions on the domain $[-1,1]^{2}$. We take $\Delta t=0.8 h^{4 / 3}$ and $t=2$ for the purpose of showing the third order accuracy. Table 2 and Table 3 list the $L_{1}$ and $L_{\infty}$ errors for the cell averages. We observe the designed order of accuracy for our scheme, justifying that the scaling limiter (3.10) does not destroy the accuracy for smooth solutions.

\subsection{Two-dimensional Burgers equation}

For piecewise smooth solutions, we consider the two-dimensional Burgers' equation:

$$
\left\{\begin{array}{l}
u_{t}+u_{x}^{2}+u_{y}^{2}=0 \\
u_{0}(x, y)=u(x, y, 0)
\end{array}\right.
$$

with periodic boundary conditions on the domain $[-2,2]^{2}$. At first, we apply the SWENO scheme to solve this equation with the initial condition $u_{0}(x, y)=\sin ^{4} \frac{\pi}{2}(x+y)$ to $t=0.05$, when the solution is still in smooth state. The numerical results for $\Delta t=0.8 h^{4 / 3}$ are reported in Table 4 and Table 5. For this nonlinear problem, the scheme still achieves the third order accuracy. In particular, the errors and $\bar{u}_{\text {min }}$ with or without the limiter are comparable.

To demonstrate the application for shock computations, we compute a classic initial problem until discontinuities occur. Fig. 4 shows the results for shocks on two kinds of meshes, both of them show that the overall solution transitions accurately. 
Table 4: Accuracy for 2D Burgers equation with scaling limiter.

\begin{tabular}{||c|cc|cc|c||}
\hline & \multicolumn{5}{|c||}{$u_{0}(x, y)=\sin ^{4}\left(\frac{\pi}{2}(x+y)\right)$} \\
\hline$N$ & $L_{1}$ error & order & $L_{\infty}$ error & order & $\bar{u}_{\min }$ \\
\hline 10 & $7.185 \times 10^{-2}$ & $\ldots$ & $8.080 \times 10^{-2}$ & $\cdots$ & $1.0 \times 10^{-17}$ \\
20 & $1.228 \times 10^{-3}$ & 2.1149 & $3.370 \times 10^{-2}$ & 1.1637 & $1.0 \times 10^{-17}$ \\
40 & $1.493 \times 10^{-3}$ & 2.3088 & $7.024 \times 10^{-3}$ & 2.3603 & $1.0 \times 10^{-17}$ \\
80 & $1.908 \times 10^{-4}$ & 3.0122 & $1.065 \times 10^{-3}$ & 2.7210 & $1.0 \times 10^{-17}$ \\
160 & $1.270 \times 10^{-5}$ & 2.9239 & $1.960 \times 10^{-4}$ & 2.4420 & $1.0 \times 10^{-17}$ \\
\hline
\end{tabular}

Table 5: Accuracy for 2D Burgers equation without scaling limiter.

\begin{tabular}{||c|cc|cc|c||}
\hline & \multicolumn{5}{|c||}{$u_{0}(x, y)=\sin ^{4}\left(\frac{\pi}{2}(x+y)\right)$} \\
\hline$N$ & $L_{1}$ error & order & $L_{\infty}$ error & order & $\bar{u}_{\min }$ \\
\hline 10 & $2.868 \times 10^{-2}$ & $\cdots$ & $7.185 \times 10^{-2}$ & $\cdots$ & 0.00000 \\
20 & $7.217 \times 10^{-3}$ & 1.9866 & $3.065 \times 10^{-2}$ & 1.2292 & $-6.50 \times 10^{-3}$ \\
40 & $1.456 \times 10^{-3}$ & 2.3095 & $8.411 \times 10^{-3}$ & 1.8655 & $-1.55 \times 10^{-3}$ \\
80 & $1.810 \times 10^{-4}$ & 3.0079 & $1.078 \times 10^{-3}$ & 2.9645 & $-1.95 \times 10^{-4}$ \\
160 & $2.467 \times 10^{-5}$ & 2.8746 & $1.864 \times 10^{-4}$ & 2.4658 & $-2.48 \times 10^{-5}$ \\
\hline
\end{tabular}

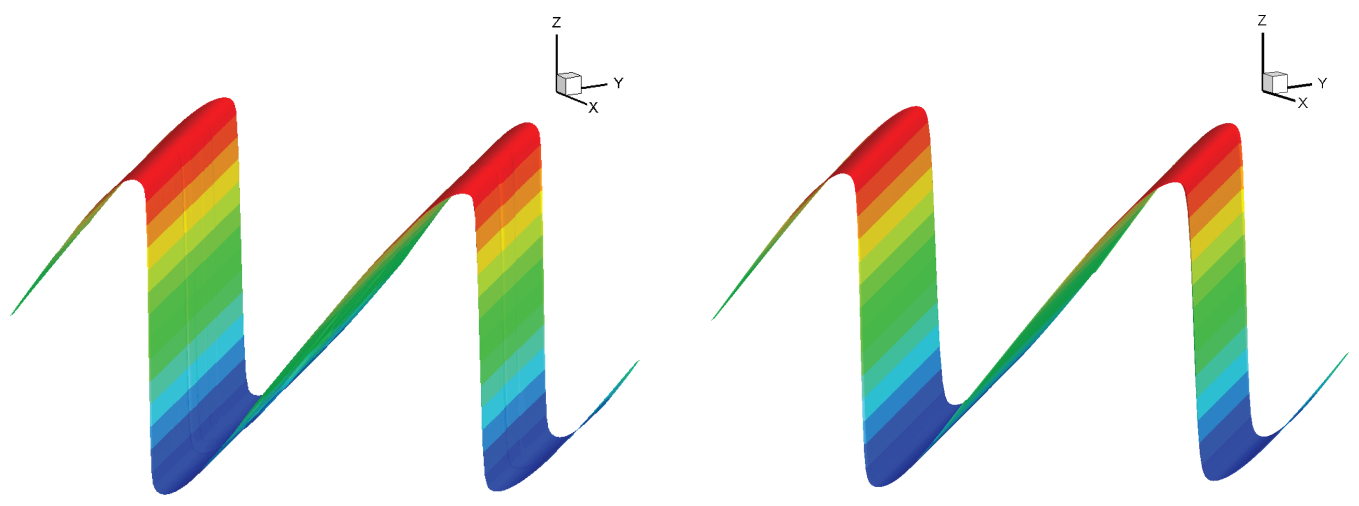

Figure 4: The numerical results with the initial condition $u(x, 0)=0.3+0.7 \sin (\pi(x+y))$ at $t=0.3$. The SWENO scheme on the non-uniform mesh (left) and the uniform mesh (right) with 25600 vertices: 3-D view.

\subsection{Two-dimensional vortex evolution problem}

The above tests demonstrate the accuracy and stability of the method for twodimensional benchmark problems. For a more realistic problem, we consider the twodimensional vortex evolution problem for the compressible Euler equations:

$$
\xi_{t}+f(\xi)_{x}+g(\xi)_{y}=0
$$


Table 6: Accuracy for 2D vortex equations without scaling limiter.

\begin{tabular}{||c|cc|cc||}
\hline$N$ & $L_{1}$ error & order & $L_{\infty}$ error & order \\
\hline 20 & $8.698 \times 10^{-3}$ & $\cdots$ & $5.002 \times 10^{-2}$ & $\cdots$ \\
40 & $4.110 \times 10^{-4}$ & 4.4025 & $8.670 \times 10^{-3}$ & 2.5285 \\
80 & $2.000 \times 10^{-5}$ & 4.3619 & $6.220 \times 10^{-4}$ & 3.8011 \\
160 & $2.454 \times 10^{-6}$ & 3.0268 & $1.004 \times 10^{-5}$ & 2.6308 \\
320 & $3.142 \times 10^{-7}$ & 2.9656 & $1.491 \times 10^{-6}$ & 2.7514 \\
\hline
\end{tabular}

with

$$
\begin{aligned}
& \xi=(\rho, \rho u, \rho v, E), \\
& f(\xi)=\left(\rho u, \rho u^{2}+p, \rho u v, u(E+p)\right), \\
& g(\xi)=\left(\rho v, \rho u v, \rho v^{2}+p, u(E+p)\right),
\end{aligned}
$$

where $\rho$ is density, $(u, v)$ is velocity, $E$ is total energy, $p$ is pressure, and

$$
E=\frac{p}{\gamma-1}+\frac{1}{2} \rho\left(u^{2}+v^{2}\right)
$$

with $\gamma=1.4$. The mean flow is $\rho=1, p=1$ and $(u, v)=(1,1)$. An isentropic vortex is added to the mean flow with perturbations in $(u, v)$ and the temperature $T=p / \rho$, but there is no perturbation in the entropy $S=p / \rho^{\gamma}$ :

$$
\begin{aligned}
& (\delta u, \delta v)=\frac{\epsilon}{2 \pi} e^{0.5\left(1-r^{2}\right)}(-\bar{y}, \bar{x}), \\
& \delta T=-\frac{(\gamma-1) \epsilon^{2}}{8 \gamma \pi^{2}} e^{1-r^{2}}, \quad \delta S=0,
\end{aligned}
$$

where $(\bar{x}, \bar{y})=(x-4, y-4), r^{2}=\bar{x}^{2}+\bar{y}^{2}$, and the vortex strength $\epsilon=5$. The computational domain is taken as $[0,8]^{2}$ and periodic boundary conditions are used. The exact solution of this problem is smooth, hence it is often used as a benchmark problem to test the accuracy of numerical schemes for solving the Euler systems. The vortex with constant speed propagates along the diagonal to the mesh lines, which gives an ample opportunity for computing the exact solution.

The reconstruction procedure is applied to each component of the solution $\xi$. We first compute the solution to $t=0.2$ for the accuracy test. Table 6 shows that the results achieve the expected convergence rate. Compared with the third order WENO scheme on triangular meshes in [2], the SWENO scheme gives a high resolution for the long-time evolution of the vortex in Fig. 7.

\subsection{One-dimensional Riemann problems of Euler equations}

In this subsection, one-dimensional Riemann problems are tested to verify the high resolution of the proposed scheme in capturing wave configurations. 

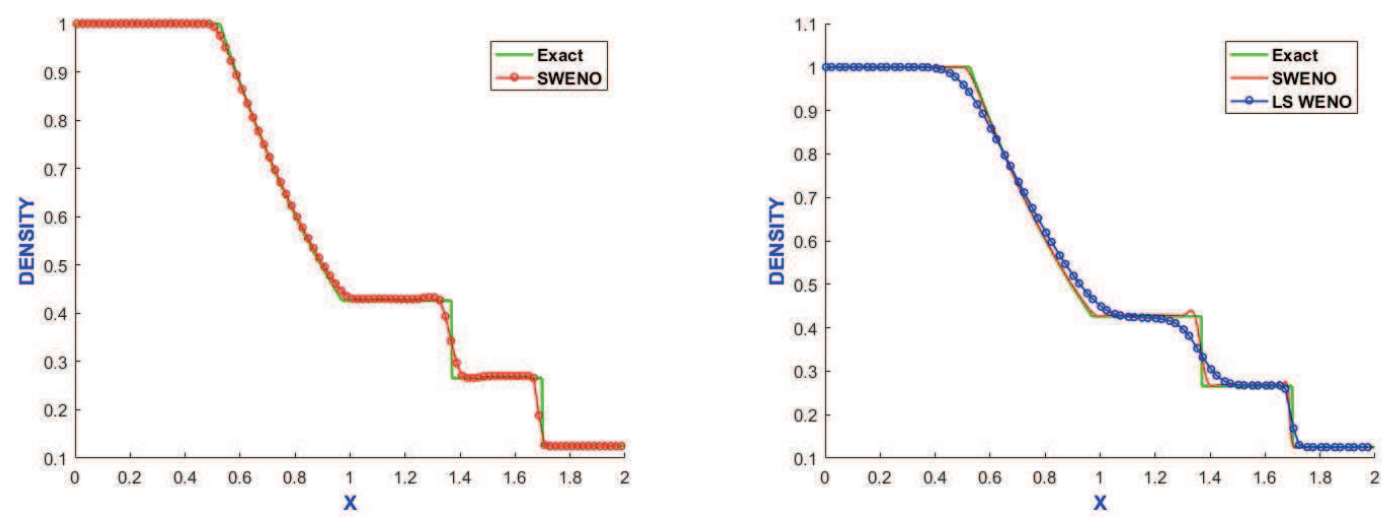

Figure 5: 1D Sod problem. The left figure is the comparison between the results of the SWENO scheme and the exact solution with the mesh size $h=0.02$ and $C F L=0.2$. The right figure is the comparison between the results of the least square WENO scheme, the SWENO scheme and the exact solution with the mesh size $h=0.01$ and $C F L=0.2$.

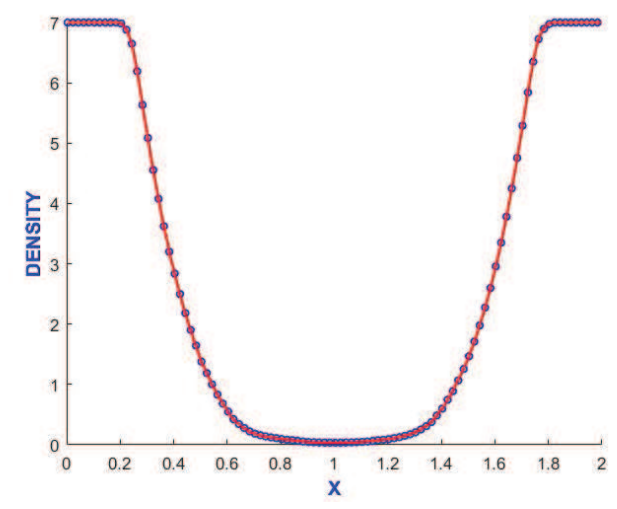

Figure 6: Double rarefaction problem.

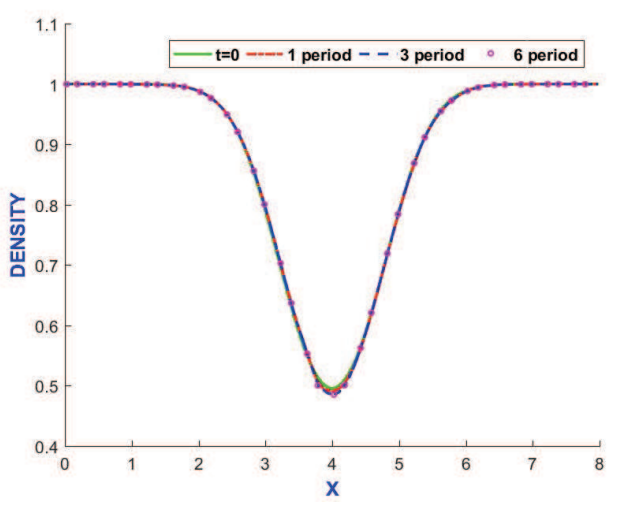

Figure 7: Vortex problem.

We consider the solution of the Euler equations (4.3) with Riemann-type initial conditions. The velocity in the $y$-direction always is zero, and periodic boundary condition is used in the $y$-direction.

The first test case is Sod's problem [17] in a domain of $[0,2] \times[0,0.4]$. The initial data are

$$
(\rho, u, p)= \begin{cases}(1,0,1), & x \leq 1 \\ (0.125,0,0.1), & x>1\end{cases}
$$

We adopt the non-uniform mesh with a triangulation of 40 vertices in the $x$-direction and 20 vertices in the $y$-direction. The plots of the density at $t=0.4$ in Fig. 5 are obtained by extracting the data along the central cut line of the triangular mesh. Obviously, the SWENO scheme has higher resolution than the least square WENO scheme when using 

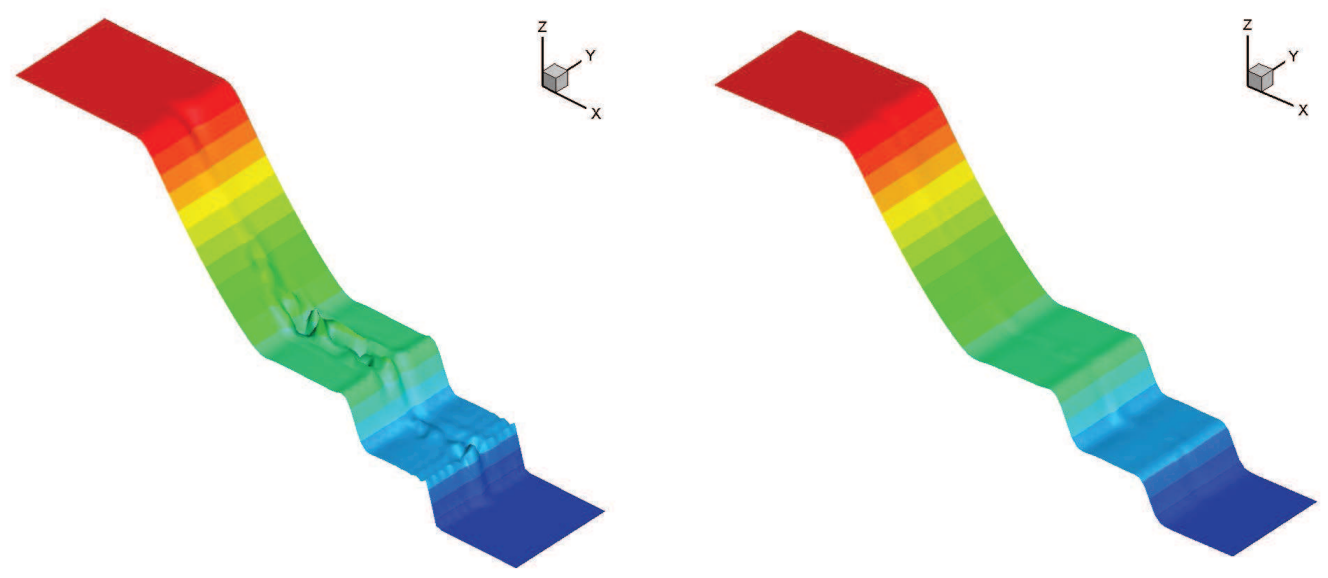

Figure 8: 1D Sod problem. The density distribution at $t=0.4$ on the low-quality non-uniform mesh. The left plot is computed by the WENO scheme [9]. The right plot is computed by the SWENO scheme: 3-D view.

the same triangular mesh and CFL condition. Fig. 8 shows the contour plots of the solutions computed by the WENO scheme in [9] and the SWENO scheme on the same low quality non-uniform mesh respectively.

The second test case is the one-dimensional low density and low pressure problem in a domain of $[-1,1] \times[0,0.4]$, which involves vacuum or near-vacuum solutions with the following initial condition:

$$
(\rho, u, p)= \begin{cases}(7,-1,0.2), & x \leq 0 \\ (7,1,0.2), & x>0\end{cases}
$$

We adopt the non-uniform mesh with a triangulation of 60 vertices in the $x$-direction and 30 vertices in the $y$-direction. The density at $t=0.6$ is shown in Fig. 6 . Our scheme gives the similar results as those presented in the literature [18]. Especially, the SWENO scheme with the scaling limiter is able to avoid spurious oscillations near the top of the rarefaction waves under a weaker CFL condition.

\subsection{Two-dimensional Buckley-Leverett equation}

The two-dimensional Buckley-Leverett (BL) equation was proposed in [19] to describe two-phase fluid flow in porous media. In particular, it is used to model the secondary oil recovery by water injection in oil reservoir. We consider the scalar equation (1.1) with the flux functions

$$
f(u)=\frac{u^{2}}{u^{2}+(1-u)^{2}}, \quad g(u)=f(u)\left(1-5\left(1-u^{2}\right)\right) .
$$

The initial data is

$$
u(x, y, 0)= \begin{cases}1, & x^{2}+y^{2}<0.5 \\ 0, & \text { otherwise }\end{cases}
$$




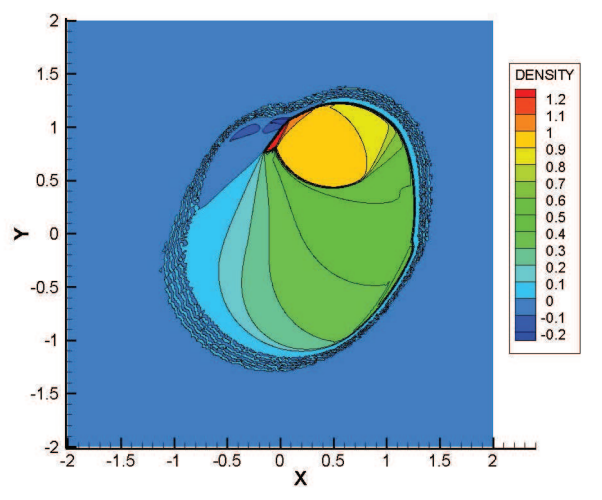

The SWENO scheme without scaling limiter

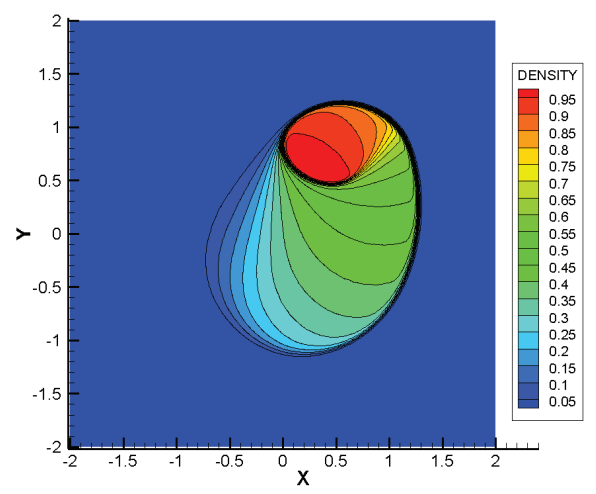

The SWENO scheme with scaling limiter

Figure 9: The solution at $t=0.5$ of the $\mathrm{BL}$ equation on the uniform mesh of $400 \times 400$ cells.

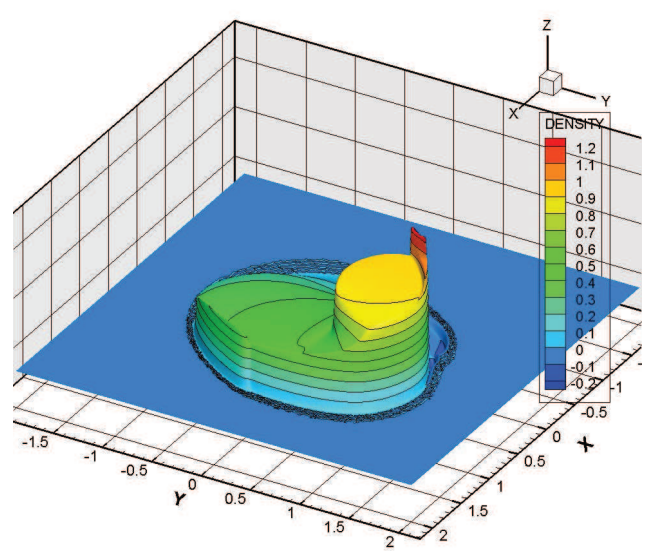

The SWENO scheme without scaling limiter

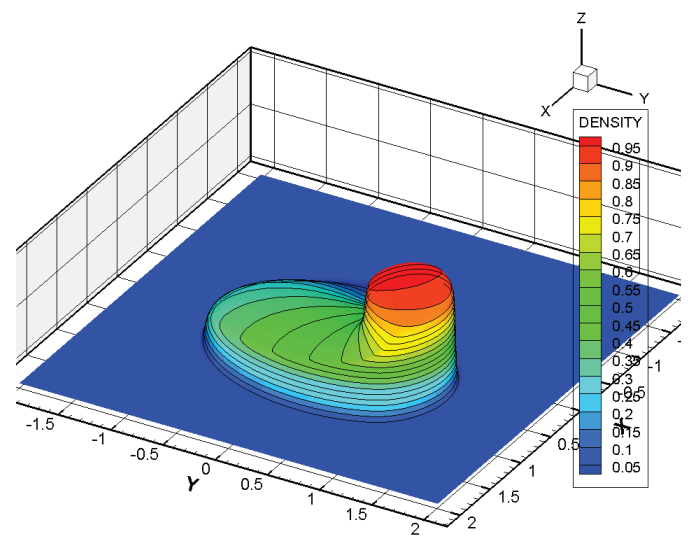

The SWENO scheme with scaling limiter

Figure 10: The solution of $2 \mathrm{D}$ BL equation at $t=0.5$ on the uniform mesh of $400 \times 400$ cells: $3-\mathrm{D}$ view.

computed in the domain $[-2,2]^{2}$. This example is taken from Karlsen et al. [20], whose exact solution is unknown. The potential negative density problem may emerge when high order WENO schemes are applied to solve it. Fig. 9 and Fig. 10 show the numerical solutions calculated by the SWENO scheme. In contradistinction to the solutions without the scaling limiter, the solutions with the scaling limiter successfully preserve the minimum and maximum of the initial data and show smoother structures.

\subsection{The kinematic frontogenesis problem}

This test problem [22] is important in meteorology where it represents a simplified effect which takes place in the atmosphere. It always is used to test the ability of the schemes 


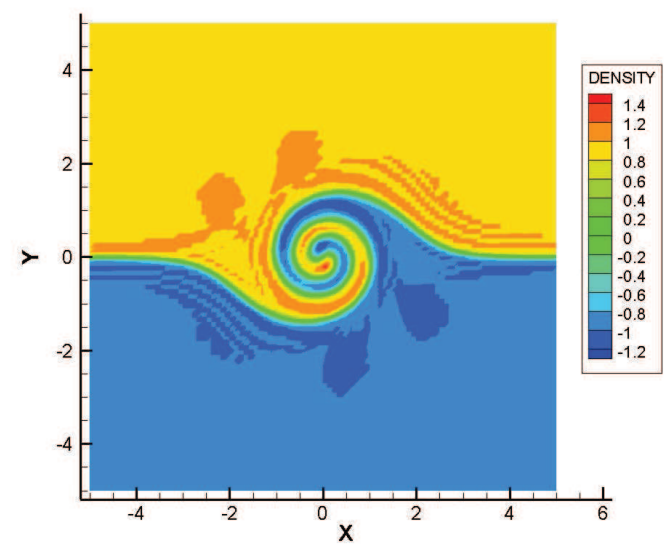

The SWENO scheme without scaling limiter

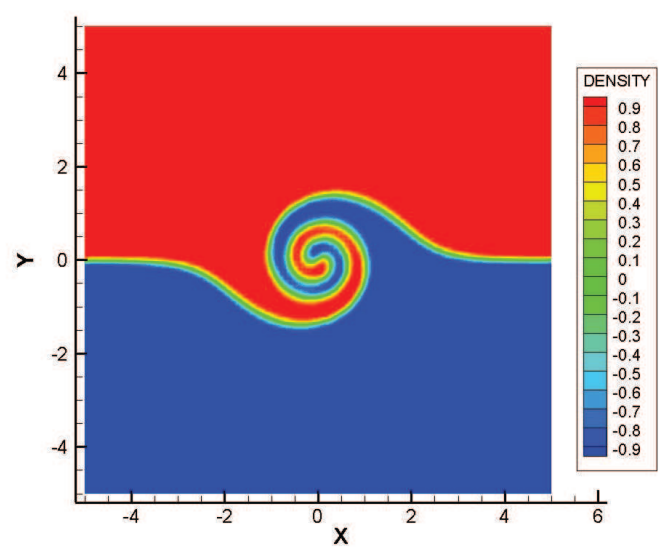

The SWENO scheme with scaling limiter

Figure 11: The solution of the kinematic frontogenesis problem on the uniform mesh of $100 \times 100$ cells.

to handle moving discontinuities in two space dimensions. We remark that a number of high order schemes have been reported to fail for this test problem, including the WENO scheme in [9]. We solve the two-dimensional linear equation with variable coefficients

$$
q_{t}+(u(x, y) q)_{x}+(v(x, y) q)_{y}=0,
$$

where $(u, v)$ is a steady divergence-free velocity field:

$$
\begin{array}{ll}
u=-y \omega(r), \quad v=x \omega(r), & \omega(r)=\frac{1}{r} U_{T}(r), \quad r^{2}=x^{2}+y^{2}, \\
U_{T}(r)=U_{\max } \operatorname{sech}^{2}(r) \tanh (r), & U_{\max }=2.5980762,
\end{array}
$$

with the initial condition defined on $[-5,5]^{2}$ :

$$
q(x, y, 0)= \begin{cases}-1, & y<0 \\ 1, & y \geq 0\end{cases}
$$

The exact solution is given by [23]:

$$
q(x, y, t)=q_{0}(y \cos (\omega t)-x \sin (\omega t))
$$

and represents a rotation of the initial discontinuous distribution around the origin with a variable angular velocity $\omega(r)$. We note that as time evolves, the solution will eventually develop scales which are beyond the resolution of the computational mesh. We compute the numerical solution to $t=4$ using both the uniform and non-uniform meshes. Observing the results shown in Fig. 11, it is obvious that the solution with the scaling limiter displays a more seared comparison with the other one. Fig. 12 shows one-dimensional 


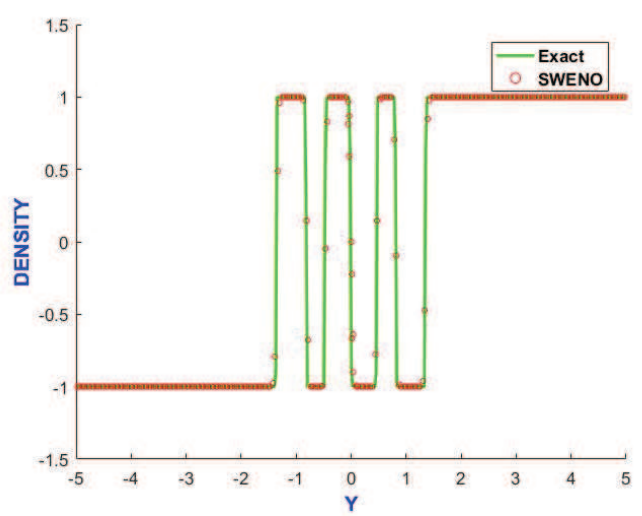

The non-uniform mesh of $400 \times 400$ cells

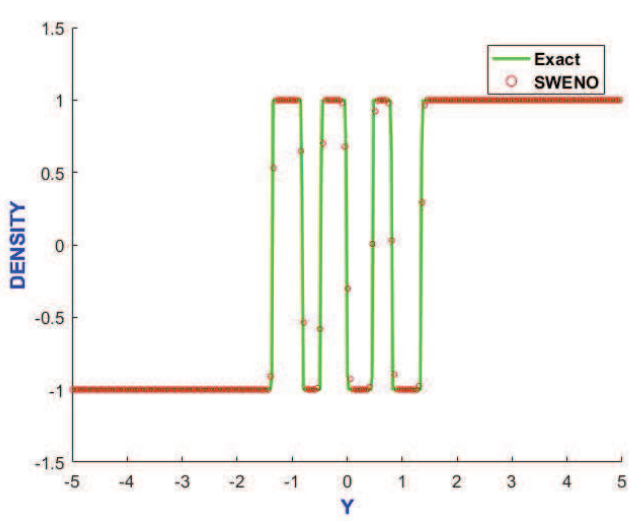

The uniform mesh of $400 \times 400$ cells

Figure 12: The solution of the kinematic frontogenesis problem computed by the SWENO scheme with scaling limiter on different triangular meshes.

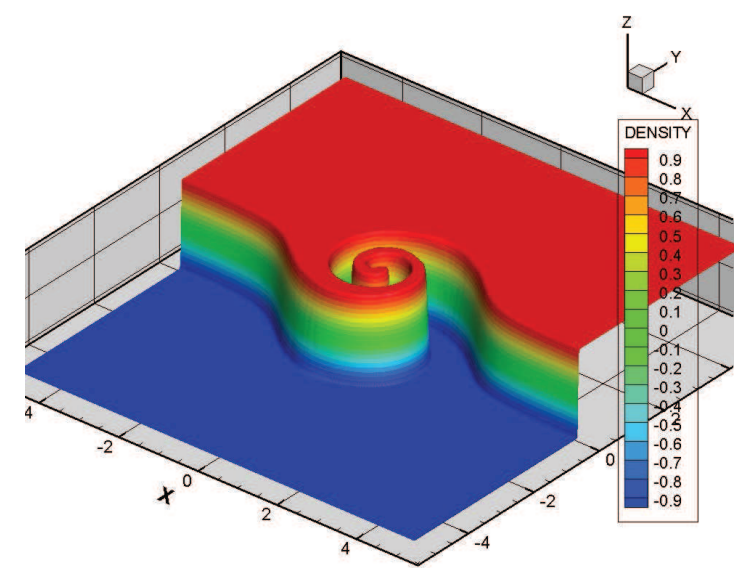

The SWENO scheme with scaling limiter

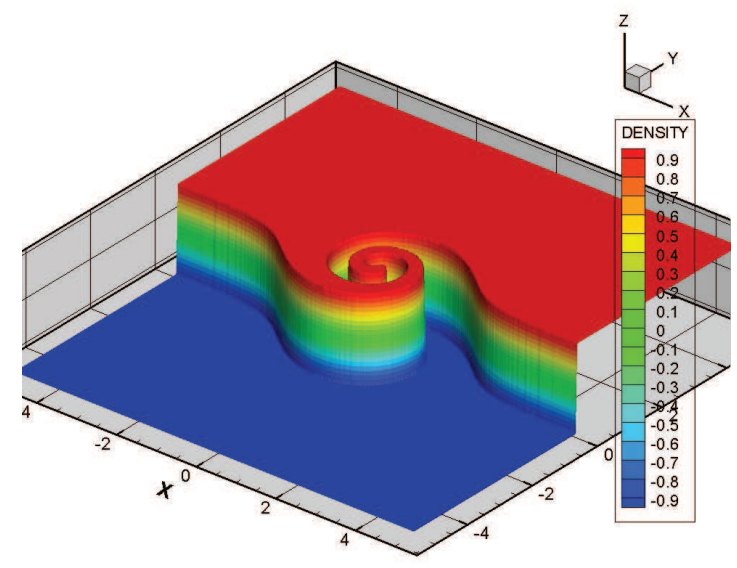

The exact solution

Figure 13: The solution of the kinematic frontogenesis problem on the non-uniform mesh of $400 \times 400$ cells: 3-D view.

cuts along the $y$ axis for $-5 \leq y \leq 5$. In all plots, the solid line corresponds to the point-wise values of the exact solution whereas symbols correspond to the numerical solution calculated by the proposed scheme on different meshes. We can observe delicate features of the solution correctly without oscillations. More details about the essentially non-oscillatory behaviors of the numerical results are clearly shown in Fig. 13. The problem has been solved by [24] using a sixth order ADER-FV scheme on triangular meshes. The comparison of their results indicates that the SWENO scheme achieves a higher resolution with the same triangle length. 


\subsection{Double mach reflection}

We solve the Euler system (4.3) on the domain $[0,3.1] \times[0,1]$. A reflecting wall lies at the bottom of the domain starting from $x=\frac{1}{6}$. Initially a right-moving Mach 10 shock is located at $x=\frac{1}{6}, y=0$ making a $60^{\circ}$ angle with the $x$-axis. The reflective boundary condition is used at the wall, while for the rest of the bottom boundary (the part from $x=0$ to $x=\frac{1}{6}$ ), the exact postshock condition is imposed. At the top boundary, the flow values are set to describe the exact motion of the Mach 10 shock. We calculate this example with the non-uniform mesh $h=\frac{1}{100}$ displayed in Fig. 14. Fig. 15 shows the numerical result for region $[0,3] \times[0,1]$ at $t=0.2$. The density plot with 35 equally spaced contour lines from 1.5 to 21.5 indicates that the proposed approach captures the complicated flow structure successfully.

\section{Concluding remarks}

In this paper, we present the reconstruction of the SWENO scheme on triangular meshes, in the context of finite volume method for solving hyperbolic conservation laws. The major novelty of the SWENO scheme is the reconstruction technique which is able to handle

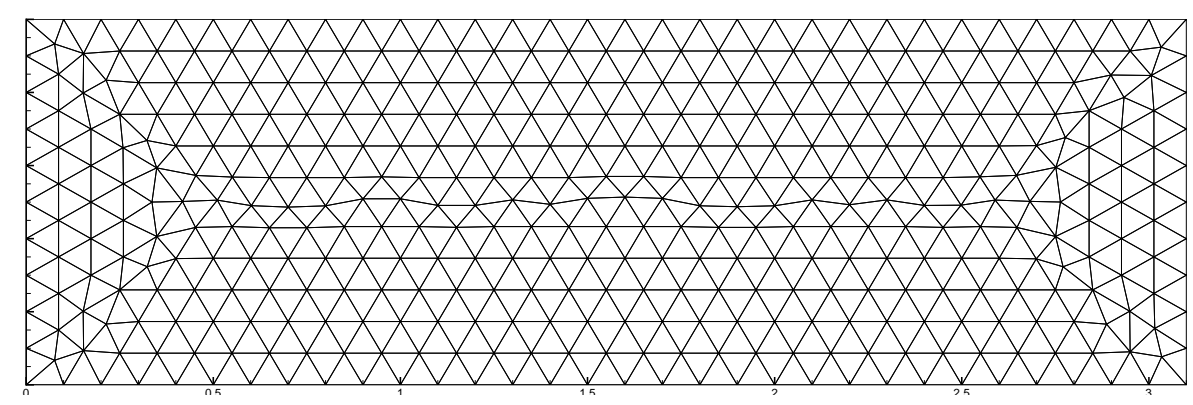

Figure 14: The non-uniform mesh for the double mach reflection.

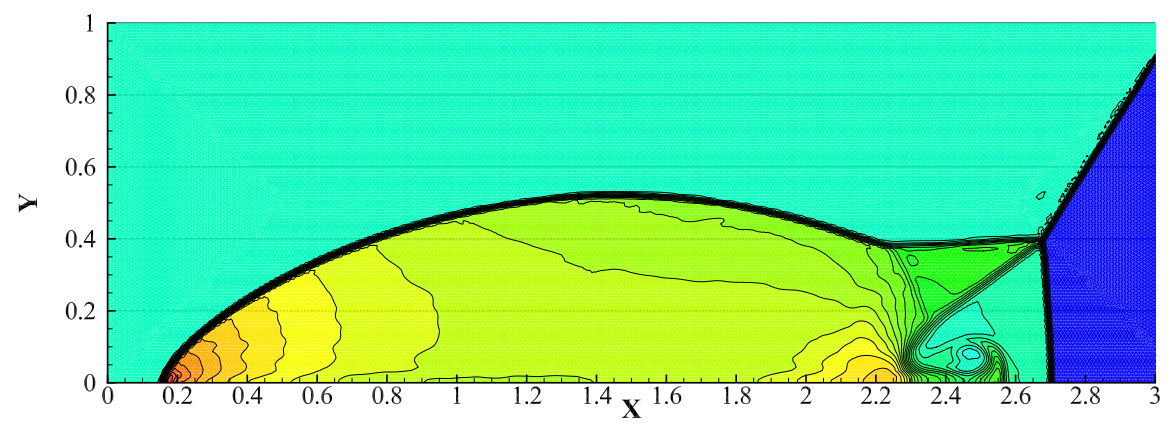

Figure 15: The numerical solution of the double mach reflection with the non-uniform mesh $h=\frac{1}{100}$. 
the moving discontinuities with less computations in two space dimensions. Besides, the modified LU decomposition and the scaling technique are adopted to implement the ill-conditioned matrix, which improve the adaptability of the SWENO scheme to low quality meshes. The scaling limiter is applied to the SWENO scheme at each stage of the third order Runge-Kutta time method. Both the accuracy and MPP property are verified theoretically and numerically with suitable CFL conditions. Finally, the two-dimensional BL equation, the kinematic frontogenesis problem and the double mach reflection show the advantages of the SWENO scheme when solving very complex hyperbolic systems in multi-phase flows, magnetohydrodynamics and general relativity.

\section{Acknowledgements}

This work was supported by the National Natural Science Foundation of China (Grant Nos. 11501570, 91530106 and 11571366), Research Fund of NUDT (Grant Nos. JC15-02-02, ZK16-03-53), and the fund from HPCL.

\section{References}

[1] ABgRAll RÉMi, On essentially non-oscillatory schemes on unstructured meshes: analysis and implementation, J. Comput. Phys., 114(1) (1994), pp. 45-58.

[2] C. Hu AND C. W. SHU, Weighted essentially non-oscillatory schemes on triangular meshes, J. Comput. Phys., 150(1) (1999), pp. 97-127.

[3] HASELBACHER, A Weno reconstruction algorithm for unstructured Grids based on explicit stencil construction, 43rd AIAA Aerospace Sciences Meeting and Exhibit, (2005), pp. 879.

[4] W. R. WOLF AND J. L. F. AZEVEDO, High-order ENO and WENO schemes for unstructured grids, Int. J. Numer. Methods Fluids, 55(10) (2007), pp. 917-943.

[5] Y. LIU AND Y. T. ZHANG, A robust reconstruction for unstructured WENO schemes, J. Sci. Comput., 54(2) (2013), pp. 603-621.

[6] H. LIU AND X. JiAO, WLS-ENO: Weighted-least-squares based essentially non-oscillatory schemes for finite volume methods on unstructured meshes, J. Comput. Phys., 314 (2016), pp. 749-773.

[7] FRIEDRICH AND OLIVER, Weighted essentially non-oscillatory schemes for the interpolation of mean values on unstructured grids, J. Comput. Phys., 144(1) (1998), pp. 194-212.

[8] Y. T. ZHANG AND C. W. SHU, High-order WENO schemes for Hamilton-Jacobi equations on triangular meshes, SIAM J. Sci. Comput., 24(3) (2003), pp. 1005-1030.

[9] S. H. SONG AND M. Z. CHEN, Third order accurate large-particle finite volume method on unstructured triangular meshes, SIAM J. Sci. Comput., 23(5) (2001), pp. 1456-1463.

[10] X. ZHANG AND C. W. SHU, Maximum-principle-satisfying and positivity-preserving high-order schemes for conservation laws: survey and new developments, Proceedings Math. Phys. Eng. Sci., 467(2134) (2011), pp. 2752-2776.

[11] X. ZHANG, Y. XIA AND C. W. SHU, Maximum-principle-satisfying and positivity-preserving high order discontinuous Galerkin Schemes for conservation laws on triangular meshes, J. Sci. Comput., 50(1) (2012), pp. 29-62.

[12] Y. HA, C. L. GARDNER, A. GELB AND C. W. SHU, Numerical simulation of high Mach number astrophysical jets with radiative cooling, J. Sci. Comput., 24(1) (2005), pp. 29-44. 
[13] A. J. Christlieb, Y. LIU, Q. TANG AND Z. XU, High order parametrized maximum-principlepreserving and positivity-preserving WENO schemes on unstructured meshes, J. Comput. Phys., 281 (2015), pp. 334-351.

[14] X. ZHANG AND C. W. SHU, Positivity-preserving high order finite difference WENO schemes for compressible Euler equations, J. Comput. Phys., 231(5) (2012), pp. 2245-2258.

[15] Y. KOBAYASHI AND T. OGITA, A fast and efficient algorithm for solving ill-conditioned linear systems, J. SIAM Lett., 7 (2015), pp. 1-4.

[16] C. W. SHU AND S. OSHER, Efficient implementation of essentially non-oscillatory shockcapturing schemes, J. Comput. Phys., 77(2) (1988), pp. 439-471.

[17] SOD AND A. GARY, A survey of several finite difference methods for systems of nonlinear hyperbolic conservation laws, J. Comput. Phys., 27(1) (1978), pp. 1-31.

[18] D. C. SEAL, Q. TANG, Z. XU AND A. J. CHRISTLIEB, An explicit high-order single-stage singlestep positivity-preserving finite difference WENO method for the compressible Euler equations, J. Sci. Comput., 68(1) (2016), pp. 171-190.

[19] S. E. BuCKLEY AND M. C. LEVERETT, Mechanism of fluid displacement in sands, Trans. AIME, 146(1) (1942), pp. 107-116.

[20] KuRganov, AlexANDER AND E. TADMOR, New high-resolution central schemes for nonlinear conservation laws and convection-diffusion equations, J. Comput. Phys., 160(1) (2000), pp. 241282.

[21] C. W. SHu, T. A. ZANG, G. Erlebacher, D. Whitaker and S. Osher, High-order ENO schemes applied to two- and three-dimensional compressible flow, Appl. Numer. Math., 9(1) (1992), pp. 45-71.

[22] TitAREV, A. VladimiR AND E. F. TORO, Finite-volume WENO schemes for three-dimensional conservation laws, J. Comput. Phys., 201(1) (2004), pp. 238-260.

[23] DAVIESJONES AND ROBERT, Comments on a kinematic analysis of frontogenesis associated with a nondivergent vortex, J. Atmospheric Sci., 42(19) (1985), pp. 2073-2075.

[24] M. DUMBSER AND M. KÄSER, Arbitrary high order non-oscillatory finite volume schemes on unstructured meshes for linear hyperbolic systems, J. Comput. Phys., 221(2) (2007), pp. 693-723. 